\title{
Intermittent low-magnitude high-frequency vibration promotes osteogenic protein expression and inhibits osteoclastogenic cytokine secretion in osteoblasts.
}

\author{
Bin Zhao" ${ }^{1 \#, ~ R u i x i n ~} \mathrm{Li}^{2 \#}$, Shihuan Cheng ${ }^{3}$, Xin Dong ${ }^{4}$, Lei Tan', Yuying Chen ${ }^{5}$, Baohui Hao', Dong \\ $\mathrm{Zhu}^{\mathbf{1}^{*}}$ \\ ${ }^{1}$ Department of Orthopedic Traumatology, First Hospital of Jilin University, Changchun, PR China \\ ${ }^{2}$ Institute of Medical Equipment, the Academy of Military Medical Sciences, Tianjin, PR China \\ ${ }^{3}$ Department of Rehabilitation Medicine, First Hospital of Jilin University, Changchun, PR China \\ ${ }^{4}$ Medical Record Department, Chinese People's Liberation Army 461 Hospital, Changchun, PR China \\ ${ }^{5}$ The Second Affiliated Hospital of Dalian Medical University, Dalian, PR China \\ \#These authors contributed equally to this work
}

\begin{abstract}
Background: Accumulating evidence has indicated that Low-Magnitude High-Frequency Vibration (LMHFV) can promote fracture healing, and thus, holds promise as a treatment for osteoporosis. However, few studies have reported the effects of LMHFV, especially intermittent LMHFV (iLMHFV) on osteoblasts in vitro. In this study, we examined the effects of sostenuto and intermittent LMHFV (sLMHFV and iLMHFV, respectively) on MC3T3-E1 preosteoblast proliferation and production of osteogenic and osteoclastogenic proteins.

Methods: The cell proliferation was determined using MTT assay for up to $7 \mathrm{~d}$. The expression levels of the osteogenic proteins Alkaline Phosphatase (ALP), Osteocalcin (OCN), and Collagen type I (COL I), and the osteoclastogenic protein nuclear factor- $\mathrm{kB}$ ligand (RANKL) and its inhibitor Osteoprotegerin (OPG) were determined by Real time PCR and/or Western blot analysis. The ALP activity was determined using a fluorometric assay. The secretion of osteoclastogenic cytokines RANKL and M-CSF was assessed by ELISA.

Results: Our results showed that neither SLMHFV nor iLMHFV affected MC3T3-E1 cell proliferation. However, both sLMHFV and iLMHFV increased the expression of the osteogenic proteins ALP, OCN, and COL I. SLMHFV and ILMHFV also inhibited the secretion of the osteoclastogenic cytokines RANKL and M-CSF and decreased the OPG/RANKL ratio. These osteogenic and anti-osteoclastogenic effects were most profound with short iLMHFV.

Conclusion: These finding could eventually contribute to the development of potential treatment regimen for osteoporosis using LMHFV.
\end{abstract}

Keywords: Sostenuto low-magnitude high-frequency vibration, Intermittent low-magnitude high-frequency vibration, Osteoblast, Osteogenic proteins, Osteoclastogenic cytokines.

Accepted on December 1, 2017

\section{Introduction}

Bone fracture is the most common injury in traffic accidents and falls, especially in elderly patients with osteoporosis [1,2]. $5 \%-10 \%$ of all patients with bone fracture often suffer from delayed unions or non-unions, which may result in long-term disability [3]. There are about 8.9 million of osteoporosisrelated fractures each year globally, which brings a great burden to public health [2].
Bone homeostasis is an intricate balance between bone formation by osteoblasts and bone resorption by osteoclasts. During the process of fracture healing, osteoblasts must proliferate, differentiate, and induce mineralization within bone extracellular matrix. Osteoblast differentiation plays a vital role during bone fracture healing. This process is characterized by increased expression of osteogenic proteins such as Alkaline Phosphatase (ALP), Osteocalcin (OCN), and Collagen type I (COL I). Mechanical forces can affect bone formation and 
repair through regulation of osteoblast differentiation and the intricate interplay between osteoblasts and osteoclasts [4-6]. Osteoblasts regulate osteoclast differentiation by producing osteoclastogenic cytokines including Macrophage ColonyStimulating Factor (M-CSF) and nuclear factor- $\kappa \mathrm{B}$ ligand (RANKL). Mechanical forces can also inhibit the production of these osteoblast-derived osteoclastogenic cytokines, thereby slowing down the process of bone resorption [5,7-9].

Sostenuto Low-Magnitude High-Frequency Vibration (sLMHFV) in the range of 0.2-0.3 $\mathrm{g}$ and $32-37 \mathrm{~Hz}$ increases bone density and prevent bone from developing osteoporosis [10-13]. It also promotes closed femoral fracture healing [14]. In agreement with these in vivo effects, sLMHFV (0.3 g, 40 $\mathrm{Hz}, 30 \mathrm{~min} / 12 \mathrm{~h}$ ) increases osteoblast differentiation, matrix synthesis, and mineralization in vitro $[15,16]$. Interestingly, studies have also shown that intermittent LMHFV (iLMHFV) is more effective than sLMHFV in improving the biomechanical properties and microstructure of bone tissue in ovariectomized rats [17]. However, there are few reports on the effects of iLMHFV on osteoblasts in vitro.

In this study, we examined the effects of sLMHFV and iLMHFV on osteoblast proliferation, osteogenic protein expression, and osteoclastogenic cytokine production in vitro. We found that iLMHFV was more effective than sLMHFV in promoting osteogenic protein expression and inhibiting osteoclastogenic cytokine secretion. Moreover, short iLMHFV can provide optimal osteogenic and anti-osteoclastogenic effects on osteoblasts.

\section{Materials and Methods}

\section{MC3T3-E1 cell culture}

The mouse preosteoblast MC3T3-E1 cell line was obtained from the Cell Resource Center IBMS CAMS/PUMS (Beijing, China). The cells were cultured in modified Eagles minimum essential medium ( $\alpha$-MEM; Invitrogen, USA) supplemented with $10 \%$ Fetal Bovine Serum (FBS) and $1 \%$ penicillinstreptomycin. The cells were maintained at $37^{\circ} \mathrm{C}, 5 \% \mathrm{CO}_{2}$ in a humidified incubator. After having reached around $90 \%$ confluence, the cells were harvested by treatment with $10 \%$ trypsin-EDTA (Sigma), seeded in six-well plates, and cultured for another $24 \mathrm{~h}$ prior to vibration treatment.

\section{LMHFV treatment}

The MC3T3-E1 cells were subjected to SLMHFV or iLMHFV $(0.25 \mathrm{~g}, 35 \mathrm{~Hz})$ treatment on a six-well vibration table (DH40020) manufactured at Hebei University of Technology. For studies on osteoblast phenotypic gene expression, we set up six groups: Control, sostenuto vibration $0.5 \mathrm{~h}(\mathrm{SV} 0.5 \mathrm{~h})$, vibration $15 \mathrm{minX} 2$-interval $0.5 \mathrm{~h}$ (IV0.5 h), vibration 15 minX2-interval1 $\mathrm{h}$ (IV1 h), vibration 15 minX2-interval $2 \mathrm{~h}$ (IV2 h), vibration 15 minX2-interval $3 \mathrm{~h}$ (IV3 h), vibration 15 minX2-interval $6 \mathrm{~h}$ (IV6 h). Untreated cells were used as negative control. For studies on osteoblast proliferation, the cells were treated each day with sostenuto vibration or intermittent vibration as above for a total of $1,3,5$, or $7 \mathrm{~d}$. Untreated cells were used as negative control.

\section{The MTT assay}

The effects of iLMHFV and sLMHFV treatment on MC3T3E1 cell proliferation were determined using the Methyl Thiazolyltetrazolium (MTT) assay. Briefly, MC3T3-E1 cells were cultured for another $24 \mathrm{~h}$ after vibration treatment. MTT (Promega) solution $(5 \mathrm{mg} / \mathrm{ml}, 1 \mathrm{ml} /$ well) was added, and the cells were incubated for $4 \mathrm{~h}$. After that, the supernatant was removed, and $2 \mathrm{ml}$ Dimethyl Sulfoxide (DMSO) was added to dissolve the formazan crystals formed. Absorbance at $490 \mathrm{~nm}$ was recorded by a scanning multiwell spectrophotometer (iMark, Bio-Rad, US). Each experiment was performed in triplicate.

\section{Alkaline phosphatase (ALP) activity analysis}

After vibration treatment, MC3T3-E1 cells were harvested and lysed in lysis buffer (10 mM HEPES, $250 \mathrm{mM}$ sucrose, $5 \mathrm{mM}$ Tris-HCl, $0.1 \%$ TritonX-100, $\mathrm{pH} 7.5$ ) with short bursts of sonication. The cell lysates were centrifuged at $4{ }^{\circ} \mathrm{C}$ overnight. The ALP activity in the supernatant was determined using a fluorometric detection kit (Nanjing KeyGENBioTECH Co. Ltd, Nanjing, China). Relative ALP units were normalized to sample protein concentration. Each experiment was performed in triplicate.

\section{RNA extraction and real time PCR}

MC3T3-E1 cells were harvested after vibration treatment. Total RNA was extracted using Trizol reagent (Beijing Biosntech Co. Ltd, Beijing China). RNA (10 $\mu$ l) was reverse transcribed using random hexamer primers and transcriptase from the Superscript III Reverse Transcriptase Kit (TIANGEN Biotech (Beijing) Co., Ltd Beijing China). Real time PCR was carried out on an IQ5 Bio-rad Real-time PCR System following manufacturer's instructions. Primer sequences are shown in Table 1. Relative mRNA expression was calculated using the ${ }^{\Delta \Delta} \mathrm{C}(\mathrm{t})$ method [18] and normalized to $\beta$-actin. Each experiment was performed in triplicate.

\section{Western blot analysis}

MC3T3-E1 cells were harvested after vibration treatment. Total cell lysates were prepared in RIPA lysis buffer containing protease and phosphatase inhibitors. Nuclear lysates were prepared as previously described [19]. Protein concentration was determined using the BCA method. Samples $(20 \mu \mathrm{g}$ proteins) were separated by SDS-PAGE and transferred to PVDF membranes (Millipore, USA). After blocking in 5\% skim milk, the membranes were incubated overnight with antiOCN and anti-Col I antibodies (Santa Cruz, USA), respectively, at $4^{\circ} \mathrm{C}$. After washing, the membranes were incubated with horseradish peroxidase-conjugated secondary antibody. The protein bands were visualized using enhanced chemiluminescence detection kit (TIANGEN BIOTECH CO., LTD, Beijing, China). Relative protein expression was 
Intermittent low-magnitude high-frequency vibration promotes osteogenic protein expression and inhibits osteoclastogenic cytokine secretion in osteoblasts

normalized to GAPDH and presented as fold of control. Each experiment was performed in triplicate.

\section{ELISA}

Secretion of osteoclastogenic cytokines RANKL and M-CSF were assessed by enzyme-linked immunosorbent assay(ELISA).After vibration treatment, RANKL and M-CSF levels in the MC3T3-E1 cell culture medium were determined using RANKL and M-CSF ELISA kits (Cloud-Clone Corp., USA), respectively, following manufacturer's instructions. Data were normalized to total protein content. Each experiment was performed in triplicate.

\section{Statistical analysis}

Statistical analysis was performed using SPSS13.0 software. All results are presented as mean \pm S.D. (Standard Deviation). Data were interpreted for normal distribution using ShapiroWild test. Multiple comparisons were analysed using one-way ANOVA followed by Duncan's multiple range test. Differences with a P-value less than 0.05 were considered statistically significant.

\section{Results}

\section{Neither LMHFV nor iLMHFV affects MC3T3-E1 cell proliferation}

First, we examined the effects of sLMHFV and iLMHFV on MC3T3-E1 cell proliferation for up to $7 \mathrm{~d}$. Each day, the cells were treated with sostenuto vibration for $0.5 \mathrm{~h}(\mathrm{SV} 0.5 \mathrm{~h})$ or vibration $15 \mathrm{minX} 2$-interval $0.5 \mathrm{~h}$ (IV0.5 h), vibration 15 minX2-intervallh (IV1 h), vibration 15 minX2-interval $2 \mathrm{~h}$ (IV2 h), vibration 15 minX2-interval $3 \mathrm{~h}$ (IV3 h), vibration 15 minX2-interval $6 \mathrm{~h}$ (IV6 h).Our MTT assay results indicated that's LMHFV or iLMHFV-treated cells proliferated at the same rate as the untreated cells throughout the experiment (Figure 1). Thus, sLMHFV and iLMHFV does not have obvient effects on osteoblast proliferation.

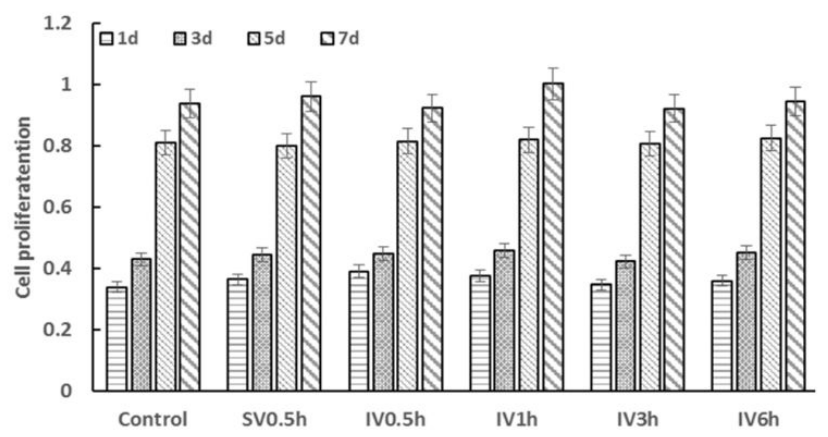

Figure 1. Neither sLMHFV nor iLMHFV affects MC3T3-E1 cell proliferation. MC3T3-E1 cells were treated each day with $S L M H F V$ for $0.5 \mathrm{~h}$ or iLMHFV for $0.5 \mathrm{~h}, 1 \mathrm{~h}, 3 \mathrm{~h}$, or $6 \mathrm{~h}$, for a total of $1 \mathrm{~d}, 3 \mathrm{~d}$, $5 d$, and $7 d$, respectively. Cell proliferation was determined by the MTT assay. $n=3$.

\section{SLMHFV and iLMHFV increase ALP $\mathrm{mRNA}$ expression level and enzymatic activity in MC3T3-E1 cells}

Alkaline Phosphatase (ALP), a key enzyme that provides high concentrations of phosphate at the site of mineral deposition, is a key factor of osteoblast early differentiation [20]. We examined the effects of sLMHFV and iLMHFV on ALP mRNA expression level and enzymatic activity in MC3T3-E1 cells. The cells were treated with sostenuto vibration for $0.5 \mathrm{~h}$ or intermittent vibration for $0.5 \mathrm{~h}, 1 \mathrm{~h}, 3 \mathrm{~h}$, or $6 \mathrm{~h}$. The ALP mRNA levels and enzymatic activity were determined by real time PCR and a fluorometric ALP assay, respectively. As shown in Figure 2A, both sLMHFV and iLMHFV-treated cells displayed significantly higher ALP mRNA levels compared to untreated cells. Interestingly, $0.5 \mathrm{~h}$ or $1 \mathrm{~h}$ intermittent vibration was significantly more effective in increasing ALP expression than $0.5 \mathrm{~h}$ constant vibration with $0.5 \mathrm{~h}$ group $110 \%$ more and $1 \mathrm{~h}$ group $20 \%$ more, respectively. Moreover, $0.5 \mathrm{~h}$ intermittent vibration appeared to be optimal, with longer vibration being less effective. Consistent with the mRNA expression results, the ALP enzymatic activity was significantly enhanced by both sLMHFV and iLMHFV treatment, with iLMHFV demonstrating more robust effects (Figure 2B).
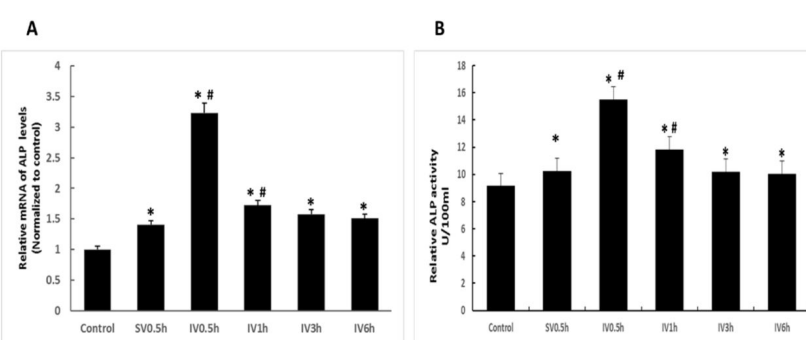

Figure 2. Both sLMHFV and iLMHFV significantly increase ALP mRNA expression and activity. MC3T3-E1 cells were treated with sLMHFV for $0.5 \mathrm{~h}$ or $i L M H F V$ for $0.5 \mathrm{~h}, 1 \mathrm{~h}, 3 \mathrm{~h}$, or $6 \mathrm{~h}$. The ALP $m R N A$ expression $(A)$ and enzymatic activity $(B)$ were determined by real time PCR and a fluorometric ALP assay, respectively. $n=3$, ${ }^{*} P<0.05$ versus Control; ${ }^{\#} P<0.05$ versus $S V 0.5 \mathrm{~h}$.

\section{SLMHFV and ILMHFV induce OCN and COL I expression in MC3T3-E1 cells}

We subsequently examined the effects of sLMHFV and iLMHFV on the mRNA and protein expression level of the osteogenic proteins OCN and COLI, the key markers of osteoblast differentiation at later stage. OCN, a protein secreted solely by osteoblasts, is present in bone mineralization and calcium ion homeostasis. COLI is the main component of bone ECM (Extra Cellular Matrix). MC3T3-E1 cells were treated with sostenuto vibration for $0.5 \mathrm{~h}$ or intermittent vibration for $0.5 \mathrm{~h}, 1 \mathrm{~h}, 3 \mathrm{~h}$, or $6 \mathrm{~h}$. The OCN and COL I mRNA and protein levels were determined by real time PCR and Western blot analysis, respectively. As shown in Figures $3 \mathrm{~A}$ and $4 \mathrm{~A}$, the mRNA levels of OCN and COLI in MC3T3-E1 cells significantly increased after SLMHFV or iLMHFV treatment. Moreover, intermittent vibration (OCN: $0.5 \mathrm{~h}, 1 \mathrm{~h}$, or $3 \mathrm{~h}, \mathrm{COL}$ I: $0.5 \mathrm{~h})$ was superior to constant vibration $(0.5 \mathrm{~h})$ in inducing 
these osteogenic proteins. Consistent with these mRNA results, sLMHFV and iLMHFV treatment significantly elevated OCN and COL I protein levels in MC3T3-E1 cells, with iLMHFV demonstrating more robust effects (Figures $3 \mathrm{~B}$ and $3 \mathrm{C}$ and $4 \mathrm{~B}$ and $4 C$ ). Similar to what was observed with ALP, the optimal effect on OCN and COL I was obtained with $0.5 \mathrm{~h}$ intermittent vibration.

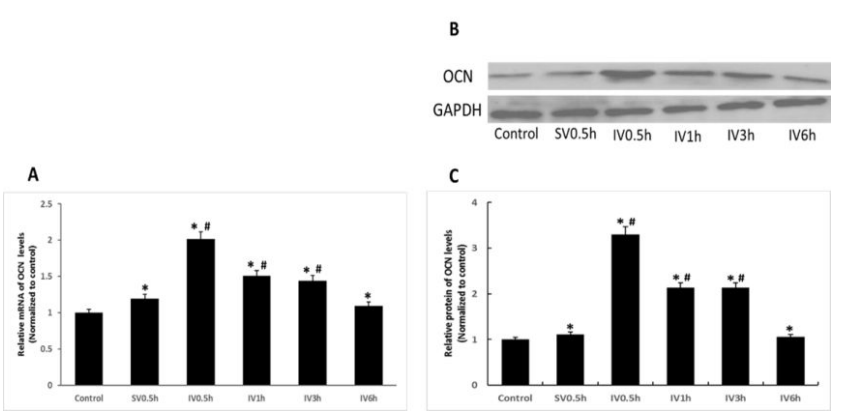

Figure 3. Both $s L M H F V$ and $i L M H F V$ significantly increase OCN mRNA and protein expression. MC3T3-E1 cells were treated with sLMHFV for $0.5 \mathrm{~h}$ or iLMHFV for $0.5 \mathrm{~h}, 1 \mathrm{~h}, 3 \mathrm{~h}$, or $6 \mathrm{~h}$. The OCN $m R N A(A)$ and protein expression ( $B$ and $C$ ) were determined by real time PCR and Western blot analysis, respectively. $n=3,{ }^{*} P<0.05$ versus control, ${ }^{\#} P<0.05$ versus $S V 0.5 \mathrm{~h}$.

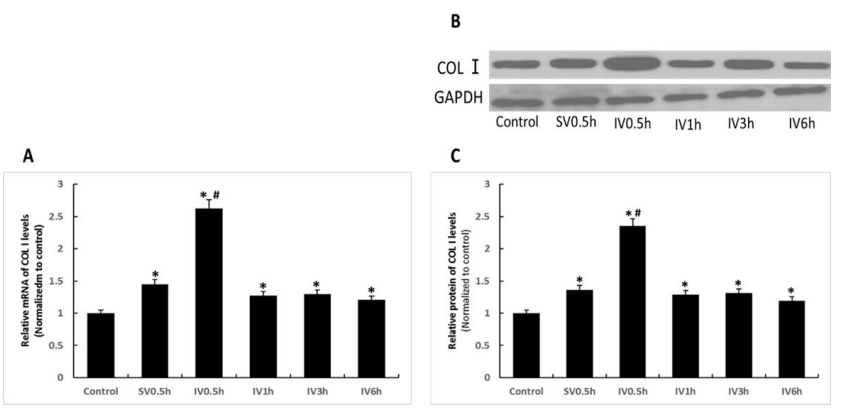

Figure 4. Both sLMHFV and iLMHFV significantly increase COL I mRNA and protein expression. MC3T3-E1 cells were treated with sLMHFV for $0.5 \mathrm{~h}$ or $\mathrm{LLMHFV}$ for $0.5 \mathrm{~h}, 1 \mathrm{~h}, 3 \mathrm{~h}$, or $6 \mathrm{~h}$. The COL $\mathrm{I}$ $m R N A(A)$ and protein expression ( $B$ and $C$ ) were determined by real time $P C R$ and Western blot analysis, respectively. $n=3,{ }^{*} P<0.05$ versus control, ${ }^{\#} P<0.05$ versus $S V 0.5 \mathrm{~h}$.

\section{SLMHFV and iLMHFV increase the OPG/RANKL ratio in MC3T3-E1 cells}

Osteoblasts regulate osteoclast differentiation through production of M-CSF, RANKL, and OPG. OPG is a decoy receptor for RANKL, which binds to RANKL blocks the RANKL-RANK interaction between osteoblast and osteoclast precursors, thereby inhibiting osteoclastogenesis. The ratio of OPG/RANKL determines osteoclast differentiation and consequently the bone resorption rate [14]. We examined the effects of sLMHFV and iLMHFV on the OPG/RANKL ratio in MC3T3-E1 cells. The cells were treated with sostenuto vibration for $0.5 \mathrm{~h}$ or intermittent vibration for $0.5 \mathrm{~h}, 1 \mathrm{~h}, 3 \mathrm{~h}$, or $6 \mathrm{~h}$. The OPG and RANKL mRNA levels were determined by real time PCR and the OPG/RANKL ratios were subsequently calculated. As shown in Figure 5, sLMHFV and iLMHFV treatment significantly increased the OPG/RANKL ratio in MC3T3-E1 cells, suggesting that these treatments inhibit the osteoclastogenic potential of osteoblasts. Similar to what was observed with osteogenic protein expression, iLMHFV $(0.5 \mathrm{~h}, 1 \mathrm{~h}$, or $3 \mathrm{~h})$ treatment outperformed sLMHFV $(0.5 \mathrm{~h})$ in increasing the OPG/RANKL ratio, with $0.5 \mathrm{~h}, 1 \mathrm{~h}$, and $3 \mathrm{~h}$ has $124 \%, 105 \%$, and $96 \%$ higher than SLMHFV $(0.5$ $\mathrm{h}$ ), and the optimal effects were obtained with $0.5 \mathrm{~h}$ intermittent vibration.

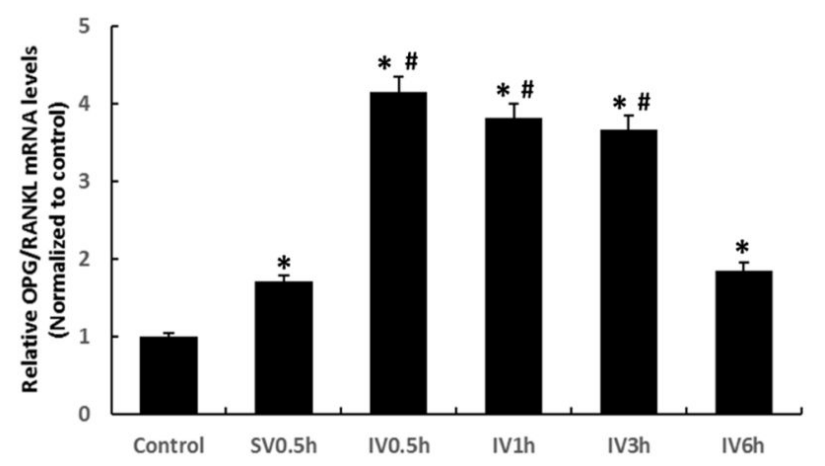

Figure 5. SLMHFV and $I L M H F V$ significantly increase the $O P G /$ RANKL ratio. MC3T3-E1 cells were treated with sostenuto vibration for $0.5 \mathrm{~h}$ or intermittent vibration for $0.5 \mathrm{~h}, 1 \mathrm{~h}, 3 \mathrm{~h}$, or $6 \mathrm{~h}$. The OPG and RANKL $m R N A$ levels were determined by real time $P C R$, and the OPG/RANKL ratios were subsequently calculated. $n=3,{ }^{*} P<0.05$ versus Control, ${ }^{\#} P<0.05$ versus $S V 0.5 \mathrm{~h}$.

\section{sLMHFV and iLMHFV inhibit M-CSF and RANKL secretion by MC3T3-E1 cells}

It has been reported that mechanical loading can suppress osteoclast activation through inhibition of the production of osteoblast-derived RANKL and M-CSF [5,7,8,14]. In this study, we examined the effects of SLMHFV and iLMHFV on M-CSF and RANKL secretion by MC3T3-E1 cells. The cells were treated with sostenuto vibration for $0.5 \mathrm{~h}$ or intermittent vibration for $0.5 \mathrm{~h}, 1 \mathrm{~h}, 3 \mathrm{~h}$, or $6 \mathrm{~h}$. The secreted RANKL and M-CSF protein in the culture medium were determined by ELISA. Our results revealed that both SLMHFV and iLMHFV treatment led to significantly decreased M-CSF and RANKL concentrations in the culture medium (Table 2), suggesting that these treatments suppress osteoblast-mediated osteoclast activation. In addition, iLMHFV $(0.5 \mathrm{~h}$ or $1 \mathrm{~h})$ outperformed sLMHFV $(0.5 \mathrm{~h})$ in inhibiting M-CSF and RANKL secretion, and the optimal inhibitory effects on both cytokines were obtained with $0.5 \mathrm{~h}$ intermittent vibration.

MC3T3-E1 cells were treated with sostenuto vibration for $0.5 \mathrm{~h}$ or intermittent vibration for $0.5 \mathrm{~h}, 1 \mathrm{~h}, 3 \mathrm{~h}$, or $6 \mathrm{~h}$. The RANKL and M-CSF protein concentrations in the culture medium were determined by ELISA. $\mathrm{n}=3,{ }^{*} \mathrm{P}<0.05$ versus control, ${ }^{\#} \mathrm{P}<0.05$ versus SV0.5 h.

Table 1. Primer sequences for real time PCR.

\begin{tabular}{lll}
\hline $\begin{array}{l}\text { Gene } \\
\text { (mouse) }\end{array}$ & Forward & Reverse \\
\hline$A L P$ & GAAGGAGGCAGGATTGAC & ATCAGCAGTAACCACAGTC \\
\hline
\end{tabular}


Intermittent low-magnitude high-frequency vibration promotes osteogenic protein expression and inhibits osteoclastogenic cytokine secretion in osteoblasts

\begin{tabular}{lll}
\hline OCN & CCATCTTTCTGCTCACTCT & ACTACCTTATTGCCCTCCT \\
\hline COLI & CAGTGGCGGTTATGACTT & CTGCGGATGTTCTCAATCT \\
\hline OPG & GCCCTGACCACTCTTATAC & AACGCCCTTCCTCACACT \\
\hline
\end{tabular}

\begin{tabular}{lll}
\hline RANKL & ATCAGAAGACAGCACTCAC & GGCTTGTTTCATCCTCCT \\
\hline$\beta$-actin & GATTACTGCTCTGGCTCCT & ACTCCTGCTTGCTGATCC \\
\hline
\end{tabular}

Table 2. SLMHFV and iLMHFV inhibit M-CSF and RANKL secretion by MC3T3-E1 cells.

\begin{tabular}{|c|c|c|c|c|c|c|}
\hline Cytokines & Control & SV0.5 h & IV0.5 h & IV1 h & IV3 h & IV6 h \\
\hline RANKL (pg/ml) & $273 \pm 15$ & $263 \pm 15^{*}$ & $200 \pm 18^{* / \#}$ & $231 \pm 13^{* / \#}$ & $274 \pm 13$ & $277 \pm 17$ \\
\hline M-CSF (pg/ml) & $70.7 \pm 2.8$ & $54.1 \pm 3.6^{*}$ & $41.1 \pm 3.4^{* / \#}$ & $49.8 \pm 2.6^{* / \#}$ & $57.6 \pm 2.4^{*}$ & $60.6 \pm 2.2^{*}$ \\
\hline
\end{tabular}

\section{Discussion}

Mechanical loading has been well recognized as one of the key regulators for the mechanical and biological structure and function of bone. Bone-related cells respond to a diverse range of mechanical stimulations such as tensile stress from stretching [19,21-26], shear stress from fluid flow [27], fourpoint bending [28], and hydrostatic pressure [29]. In recent years, accumulating evidence has demonstrated that LMHFV promotes bone formation in vivo $[9,17]$. However, the effects of LMHFV on bone cells in vitro have not been comprehensively investigated.

In this study, we examined the effects of sLMHFV and iLMHFV on osteoblast proliferation, osteogenic protein expression, and osteoclastogenic cytokine production, using the MC3T3-E1 preosteoblasts as an in vitro model system. Our MTT assay results indicated that sLMHFV or iLMHFV treatment for up to $7 \mathrm{~d}$ did not cause significant changes in MC3T3-E1 cell proliferation. Past studies on the effects of LMHFV on preosteoblast proliferation have generated controversial results. LMHFV has been reported to inhibit the proliferation of human periodontal ligament stem cells and Bone Marrow-Derived Mesenchymal Stromal Cells (BMSC) [16,30]. However, LMHFV has also been reported to promote BMSC proliferation [31]. In this study, we found that MC3T3E1 cell proliferation was not affected by SLMHFV or iLMHFV under the experimental conditions tested. Interestingly, studies have shown that the effects of pressure loading on osteoblast proliferation are frequency and duration-dependent [32]. Taking all previous studies and our data into consideration, we can believe that the effects of LMHFV on preosteoblast proliferation depend highly on the cell type and vibration parameters.

Many studies have shown that mechanical stimulation promotes osteogenic differentiation and expression of ALP, COL I and OCN, proteins required for bone matrix synthesis, both in vitro and in vivo [33-35]. Specifically, LMHFV has been shown to strengthen bones and promote fracture healing in vivo $[10,12,14,15]$. In addition, sLMHFV has been reported to promote osteogenic differentiation in vitro [16]. In this study, we found that both sLMHFV and iLMHFV significantly increased the expression of ALP, COL I, and OCN in MC3T3E1 preosteoblasts, indicating that both SLMHFV and iLMHFV promote osteogenic differentiation in vitro. Intriguingly, we found that iLMHFV was more effective than sLMHFV in inducing osteoblast differentiation, suggesting that iLMHFV may have greater osteogenic effects. We speculate that osteoblasts could adapt to the stimulation, thus fail to respond as rigorous as it does at the beginning, and could not translate the mechanical stimulation to biological responses in the continuous manner. When osteoblasts are subject to an intermittent rest between stimulation, they can regain the ability and sensitivity to vibration stimulation, thus creating a biomechanical response far better than continuous stimulation.

Previous in vitro studies have shown that SLMHFV can inhibit osteoclastic differentiation and osteoclasticresorption by suppressing the production of osteocyte-derived osteoclastogenic cytokines [14,36]. In this study, we found that both SLMHFV and iLMHFV significantly decreased the level of M-CSF and RANKL secreted by MC3T3-E1 cells. sLMHFV and iLMHFV also significantly increased the OPG/ RANKL ratio, a critical regulator of osteoclast formation, activation, and survival. Thus, our results suggested that both sLMHFV and iLMHFV have anti-resorption potential in bone remodeling and homeostasis. Intriguingly, iLMHFV has more profound effect than sLMHFV in inhibiting the production of osteoclastogenic cytokines, suggesting that iLMHFV may contribute more in protecting bone tissue from osteoclastic absorption.

Taken together, our results suggested that, compared with sLMHFV, iLMHFV has more profound effect in enhancing bone formation and healing. These in vitro results are on par with previous in vivo studies, which showed that iLMHFV loading was superior to sLMHFV loading in upregulation osteogenic proteins and promoting healing in osteoporotic fractures [17,37]. Sostenuto mechanical loading may cause adaptive tolerance to the stimuli, resulting in gradually diminishing effects [38]. We believe that periodical rest between LMHFV may help maintain or even restore the sensitivity to the stimulation of cells, and thus generating greater effect towards desired function. Moreover, we found that $0.5 \mathrm{~h}$ of iLMHFV can provide optimal results, with longer treatment being less effective. These results were in agreement with previous findings that early exposure to LMHFV can positively modulate osteoporotic bone and osteoblasts; however, the beneficial effects seem to not give a consistent performance over time [39]. We believe that this is because prolonged intermittent vibration could result in similar effect as 
sostenuto vibration, which may cause fatigue of cells to mechanical stimuli, leading to desensitization over time.

Our findings could provide useful insights in the development of clinically applicable LMHFV treatment regimen to strengthen bones and promote fracture healing. Clinical trials are required to investigate the therapeutic potential of LMHFV to treat osteoporosis in human in order to harness our findings to benefit more patients.

\section{Conclusion}

In conclusion, we have shown that both sLMHFV and iLMHFV can increase the expression level of the osteogenic proteins ALP, OCN, and COL I in MC3T3-E1 preosteoblasts. sLMHFV and iLMHFV also can inhibit the secretion of osteoclastogenic cytokines M-CSF and RANKL, as well as can increase the OPN/RANKL ratio. These osteogenic and antiosteoclastogenic effects were most profound with short iLMHFV.

\section{Acknowledgments}

This study was supported by the Key Program and General Programs of the National Natural Science Foundation of China (No. 11432016, 11272134 and 11602093).

\section{Conflict of Interest Statement}

The authors declare no conflict of interest.

\section{References}

1. Ganveer GB, Tiwari RR. Injury pattern among non-fatal road traffic accident cases: a cross-sectional study in Central India. Indian J Med Sci 2005; 59: 9-12.

2. Blotman F, Cortet B, Hilliquin P, Avouac B, Allaert FA, Pouchain D, Gaudin AF, Cotté FE, El Hasnaoui A. Characterisation of patients with postmenopausal osteoporosis in French primary healthcare. Drugs Aging 2007; 24: 603-614.

3. Nelson FR, Brighton CT, Ryaby J, Simon BJ, Nielson JH, Lorich DG, Bolander M, Seelig J. Use of physical forces in bone healing. J Am Acad Orthop Surg 2003; 11: 344-354.

4. Ehrlich PJ, Lanyon LE. Mechanical strain and bone cell function: a review. Osteoporos Int 2002; 13: 688-700.

5. Rubin J, Murphy TC, Rahnert J, Song H, Nanes MS, Greenfield EM, Jo H, Fan X. Mechanical inhibition of RANKL expression is regulated by H-Ras-GTPase. J Biol Chem 2006; 281: 1412-1418.

6. Chen JH, Liu C, You L, Simmons CA. Boning up on Wolff s Law: mechanical regulation of the cells that make and maintain bone. J Biomech 2010; 43: 108-118.

7. Fan X, Rahnert JA, Murphy TC, Nanes MS, Greenfield EM, Rubin J. Response to mechanical strain in an immortalized pre-osteoblast cell is dependent on ERK1/2. J Cell Physiol 2006; 207: 454-460.
8. Ogawa T, Zhang X, Naert I, Vermaelen P, Deroose CM, Sasaki K, Duyck J. The effect of whole-body vibration on peri-implant bone healing in rats. Clin Oral Implants Res 2011; 22: 302-307.

9. Zhang R, Gong H, Zhu D, Gao J, Fang J, Fan Y. Seven day insertion rest in whole body vibration improves multi-level bone quality in tail suspension rats. PLoS One 2014; 9: 92312.

10. Rubin C, Turner AS, Bain S, Mallinckrodt C, McLeod K. Anabolism. Low mechanical signals strengthen long bones. Nature 2001; 412: 603-604.

11. Prisby RD, Lafage-Proust MH, Malaval L, Belli A, Vico L. Effects of whole body vibration on the skeleton and other organ systems in man and animal models: what we know and what we need to know. Ageing Res Rev 2008; 7: 319-329.

12. Ozcivici E, Luu YK, Rubin CT, Judex S. Low-level vibrations retain bone marrow s osteogenic potential and augment recovery of trabecular bone during reambulation. PLoS One 2010; 5: 11178.

13. Wysocki A, Butler M, Shamliyan T, Kane RL. Whole-body vibration therapy for osteoporosis: state of the science. Ann Intern Med 2011; 155: 680-686, W206-213.

14. Zhou Y, Guan X, Liu T, Wang X, Yu M, Yang G, Wang H. Whole body vibration improves osseointegration by upregulating osteoblastic activity but down-regulating osteoblast-mediated osteoclastogenesis via ERK1/2 pathway. Bone 2015; 71: 17-24.

15. Leung KS, Shi HF, Cheung WH, Qin L, Ng WK, Tam KF, Tang N. Low-magnitude high-frequency vibration accelerates callus formation, mineralization, and fracture healing in rats. J Orthop Res 2009; 27: 458-465.

16. Zhou Y, Guan X, Zhu Z, Gao S, Zhang C, Li C, Zhou K, Hou W, Yu H. Osteogenic differentiation of bone marrowderived mesenchymal stromal cells on bone-derived scaffolds: effect of microvibration and role of ERK1/2 activation. Eur Cell Mater 2011; 22: 12-25.

17. Ma R, Zhu D, Gong H, Gu G, Huang X, Gao J, Zhang X. High-frequency and low-magnitude whole body vibration with rest days is more effective in improving skeletal micro-morphology and biomechanical properties in ovariectomised rodents. Hip Int 2012; 22: 218-226.

18. Livak KJ, Schmittgen TD. Analysis of relative gene expression data using real-time quantitative PCR and the 2(-Delta Delta C(T)) Method. Methods 2001; 25: 402-408.

19. Ziros PG, Gil AP, Georgakopoulos T, Habeos I, Kletsas D, Basdra EK, Papavassiliou AG. The bone-specific transcriptional regulator Cbfal is a target of mechanical signals in osteoblastic cells. J Biol Chem 2002; 277: 23934-23941.

20. Ducy P, Zhang R, Geoffroy V, Ridall AL, Karsenty G. Osf2/Cbfa1: a transcriptional activator of osteoblast differentiation. Cell 1997; 89: 747-754.

21. Brighton CT, Strafford B, Gross SB, Leatherwood DF, Williams JL, Pollack SR. The proliferative and synthetic response of isolated calvarial bone cells of rats to cyclic 
biaxial mechanical strain. J Bone Joint Surg Am 1991; 73: 320-331.

22. Zhuang H, Wang W, Tahernia AD, Levitz CL, Luchetti WT, Brighton CT. Mechanical strain-induced proliferation of osteoblastic cells parallels increased TGF-beta 1 mRNA. Biochem Biophys Res Commun 1996; 229: 449-453.

23. Matsuda N, Morita N, Matsuda K, Watanabe M. Proliferation and differentiation of human osteoblastic cells associated with differential activation of MAP kinases in response to epidermal growth factor, hypoxia, and mechanical stress in vitro. Biochem Biophys Res Commun 1998; 249: 350-354.

24. Cheng M, Zaman G, Rawlinson SC, Mohan S, Baylink DJ, Lanyon LE. Mechanical strain stimulates ROS cell proliferation through IGF-II and estrogen through IGF-I. J Bone Miner Res 1999; 14: 1742-1750.

25. Nagatomi J, Arulanandam BP, Metzger DW, Meunier A, Bizios R. Frequency- and duration-dependent effects of cyclic pressure on select bone cell functions. Tissue Eng 2001; 7: 717-728.

26. Weyts FA, Bosmans B, Niesing R, van Leeuwen JP, Weinans H. Mechanical control of human osteoblast apoptosis and proliferation in relation to differentiation. Calcif Tissue Int 2003; 72: 505-512.

27. Mikuni-Takagaki Y, Suzuki Y, Kawase T, Saito S. Distinct responses of different populations of bone cells to mechanical stress. Endocrinology 1996; 137: 2028-2035.

28. You J, Yellowley CE, Donahue HJ, Zhang Y, Chen Q, Jacobs CR. Substrate deformation levels associated with routine physical activity are less stimulatory to bone cells relative to loading-induced oscillatory fluid flow. J Biomech Eng 2000; 122: 387-393.

29. Kaspar D, Seidl W, Neidlinger-Wilke C, Ignatius A, Claes L. Dynamic cell stretching increases human osteoblast proliferation and CICP synthesis but decreases osteocalcin synthesis and alkaline phosphatase activity. J Biomech 2000; 33: 45-51.

30. Zhang C, Li J, Zhang L, Zhou Y, Hou W, Quan H, Li X, Chen Y, Yu H. Effects of mechanical vibration on proliferation and osteogenic differentiation of human periodontal ligament stem cells. Arch Oral Biol 2012; 57: 1395-1407.

31. Luu YK, Capilla E, Rosen CJ, Gilsanz V, Pessin JE, Judex $\mathrm{S}$, Rubin CT. Mechanical stimulation of mesenchymal stem cell proliferation and differentiation promotes osteogenesis while preventing dietary-induced obesity. J Bone Miner Res 2009; 24: 50-61.

32. Garcia-Giralt N, Nogues X, Enjuanes A, Puig J, Mellibovsky L, Bay-Jensen A, Carreras R, Balcells S, Díez-Pérez A, Grinberg D. Two new single-nucleotide polymorphisms in the COL1A1 upstream regulatory region and their relationship to bone mineral density. J Bone Miner Res 2002; 17: 384-393.

33. Tanno M, Furukawa KI, Ueyama K, Harata S, Motomura S. Uniaxial cyclic stretch induces osteogenic differentiation and synthesis of bone morphogenetic proteins of spinal ligament cells derived from patients with ossification of the posterior longitudinal ligaments. Bone 2003; 33: 475-484.

34. Guo Y, Zhang CQ, Zeng QC, Li RX, Liu L, Hao QX, Shi $\mathrm{CH}$, Zhang XZ, Yan YX. Mechanical strain promotes osteoblast ECM formation and improves its osteoinductive potential. Biomed Eng 2012; 11: 80.

35. Lu HF, Mai ZH, Xu Y, Wang W, Ai H. Mechanical loading induced expression of bone morphogenetic protein-2, alkaline phosphatase activity, and collagen synthesis in osteoblastic MC3T3-E1 cells. Chin Med J (Engl) 2012; 125: 4093-4097.

36. Lau E, Al-Dujaili S, Guenther A, Liu D, Wang L, You L. Effect of low-magnitude, high-frequency vibration on osteocytes in the regulation of osteoclasts. Bone 2010; 46: 1508-1515.

37. Li M, Wu W, Tan L, Mu D, Zhu D, Wang J, Zhao B. Lowmagnitude mechanical vibration regulates expression of osteogenic proteins in ovariectomized rats. Biochem Biophys Res Commun 2015; 465: 344-348.

38. Davies PF. Flow-mediated endothelial mechanotransduction. Physiol Rev 1995; 75: 519-560.

39. Qing F, Xie P, Liem YS, Chen Y, Chen X, Zhu X, Fan Y, Yang $\mathrm{X}$, Zhang $\mathrm{X}$. Administration duration influences the effects of low-magnitude, high-frequency vibration on ovariectomized rat bone. J Orthop Res 2016; 34: $1147-1157$.

\section{*Correspondence to}

Dong Zhu

Department of Orthopedic Traumatology Trauma

First Hospital of Jilin University

PR China 